\title{
Diversité Ligneuse et Stock de Carbone des Systèmes Agroforestiers à base de Cacaoyers à l'Est Cameroun : Cas de la Forêt d'Enseignement et de Recherche de l'Université de Dschang
}

\author{
TEMGOUA Lucie Félicité1, DONGMO William¹, NGUIMDO Vianny ${ }^{1}$, NGUENA Carrele ${ }^{1}$ \\ 1 Département de Foresterie, Faculté d'Agronomie et des Sciences Agricoles, Université de Dschang, BP 222 Dschang \\ Cameroun \\ Auteur Correspondant : TEMGOUA Lucie Félicité temgoualucie@yahoo.fr
}

Original submitted in on $14^{\text {th }}$ February 2018. Published online at www.m.elewa.org on $28^{\text {th }}$ February 2018 https://dx.doi.org/10.4314/jab.v122i1.7

\section{RESUME}

Objectifs : Au Cameroun, le cacao est produit dans des systèmes agroforestiers avec association d'arbres. Cette étude avait pour objectif d'évaluer la diversité ligneuse, les usages et les stocks de carbone des systèmes agroforestiers cacaoyers dans la forêt d'enseignement et de recherche de l'Université de Dschang (FER) à l'Est Cameroun.

Méthodologie et résultats : L'inventaire a été réalisé dans 31 parcelles de $2400 \mathrm{~m}^{2}$ et une enquête réalisée auprès des agriculteurs a permis d'identifier l'origine, les différents produits et les usages des arbres. L'estimation des stocks de carbone dans la biomasse ligneuse aérienne et souterraine a été faite à l'aide des modèles allométriques basés sur la méthode non destructive. Au total 71 espèces ligneuses appartenant à 32 familles ont été inventoriées. Les cinq espèces les plus fréquentes sont Pycnanthus angolensis, Petersianthus macrocarpus, Musanga cecropioides, Terminalia superba et Persea americana. Les arbres conservés sont les plus nombreux par rapport à ceux introduits qui sont majoritairement des fruitiers (Persea americana, Dacryodes edulis et Mangifera indica). Ces arbres associés sont pour la plupart des arbres multi-usages qui fournissent des produits alimentaires et médicinaux, du bois et participent à l'amélioration de la fertilité du sol en plus de l'ombrage fourni aux cacaoyers. La teneur moyenne en carbone des systèmes agroforestiers cacaoyers de la FER est de $107 \mathrm{tC} \mathrm{ha}^{-1}$ et dépend surtout des arbres associés qui y contribuent à plus de 95\%. Conclusions et application des résultats : Plusieurs contraintes à la plantation des arbres ont été mises en évidence et devraient être levées par une assistance technique aux agriculteurs. Les stratégies d'amélioration doivent tenir compte des espèces sollicitées par ces agriculteurs dans la recherche des meilleurs compromis entre la production cacaoyère, et les autres services rendus par les arbres.

Mots-clés : Agroforesterie, Cacaoculture, Carbone, Espèce ligneuse, Service écosystémique, Cameroun 
Tree Diversity and Carbon Stocks in Cocoa based Agroforestry Systems in Eastern Cameroon : Case Study of the Learning and Research Forest of the University of Dschang

\begin{abstract}
Objectives: In Cameroon, cocoa is produced in agroforestry systems with association of trees. The objective of this study was to evaluate the woody diversity, uses and stocks of carbon of the agroforestry cocoa systems in the teaching and research forest of the University of Dschang in eastern Cameroon.

Methodology and results: The inventory was carried out in 31 plots of $2400 \mathrm{~m}^{2}$ and a survey of farmers permitted us to identify the origin of trees, their different products and uses. An estimation of carbon stocks in above ground and underground woody biomass was made using allometric models based on non-destructive method. A total of 71 woody species belonging to 32 families were counted. The five most frequent species identified are Pycnanthus angolensis, Petersianthus macrocarpus, Musanga cecropioides, Terminalia superba, and Persea americana. Preserved trees are more abundant with respect to those introduced, which were mainly fruit trees (Persea americana, Dacryodes edulis and Mangifera indica). These associated trees are mostly multi-purpose trees that provide food, medicine and wood and contribute to the improvement of soil fertility while providing shade to cocoa. The average carbon content of the cocoa agroforestry systems is 107 tC ha-1. This amount is mainly due to the associated trees which contribute for about $95 \%$.

Conclusions and application of results: Several constraints to planting trees have been highlighted and should be taken into account via technical assistance to farmers. Strategies to improve the cocoa based agroforestry systems must take into account the tree species needed by farmers in the search for the best trade-offs between cocoa production and other services offered by trees.
\end{abstract}

Keywords: Cocoa farming, Woody species, Agroforestry, Carbon, Ecosystemic service, Cameroon

\section{INTRODUCTION}

Dans le bassin du Congo deuxième massif forestier mondial, la déforestation est grandissante. Le taux de déforestation y est passé de $0,13 \%$ dans la période 1990 à 2000 à $0,26 \%$ dans la période 2000 à 2005 (Ernst et al., 2013). Le principal facteur de cette déforestation est le défrichement des forêts au profit de l'agriculture (CARPE, 2005); et les conséquences sont la perte de la biodiversité et la réduction des services écosystémiques comme la séquestration du carbone. Dans les zones de forêt dense, les systèmes de culture comprennent les cultures itinérantes sur brûlis mais aussi les cultures commerciales, principalement le cacao (Theobroma cacao L.) (Sunderlin et al., 2000; CARPE, 2005). Les exploitations cacaoyères sont considérées comme de grands moteurs de la déforestation (Gockowski et Sonwa, 2010), puisqu'elles sont tenues par de petits agriculteurs que divers moteurs socioéconomiques poussent vers des fronts pionniers ou l'intensification (Vaast et Somariba, 2014). Le Cameroun est le cinquième pays producteur de Cacao au monde avec une production annuelle autour de 211000 tonnes (ICCO, 2017). Les principaux bassins de production du cacao sont le Centre-Sud, le SudOuest, le Littoral et l'Est où il constitue la culture de rente la plus importante pour $69 \%$ des agriculteurs (Sunderlin et al., 2000). La culture de cacao se développe généralement de façon traditionnelle sous l'ombrage naturel de la forêt (Jagoret, 2011). Elle est pratiquée majoritairement par des petits paysans dans des systèmes agroforestiers avec association des espèces ligneuses (Gockowski et al., 2010). Les agriculteurs conservent et introduisent un certain nombre d'arbres dans la parcelle (Sonwa et al., 2007, Jagoret et al., 2011; Saj et al., 2013; Saj et al.,2017). Ces arbres fournissent un ombrage aux cacaoyers et offrent également aux ménages des produits qui interviennent dans l'autosuffisance et l'équilibre alimentaire des familles (Jagoret et al., 2014a, 2014b). Avec leur structure qui s'apparente à celle des forêts, les systèmes agroforestiers (SAFs) cacaoyers participent à la conservation de la diversité ligneuse (Sonwa et al., 2007; Saj et al., 2017). La séquestration du carbone est également 
l'un des services écosystémiques non négligeables rendus par ces systèmes agroforestiers (Njomnang et al., 2011 ; Norgrove et Hauser, 2013 ; Saj et al., 2013). En générant de nombreux services écologiques, les SAFs cacaoyers sont perçus comme durables (Jagoret, 2011)] et donc éligibles au mécanisme de réduction des émissions dues à la déforestation et à la dégradation des forêts (Atangana et al., 2014). Les potentialités de conservation de la diversité ligneuse et de stockage de carbone des SAFs cacaoyers dans les bassins de production du Centre-Sud sont maintenant bien documentées (Sonwa et al., 2007 ; Jagoret et al., 2011 ; Jagoret et al., 2014a, 2014b ; Saj et al., 2017), ce qui n'est pas encore le cas dans le bassin de l'Est. Le gouvernement camerounais a classé en avril 2016, un massif forestier de 2400 ha à Belabo, dans la région de l'Est comme Forêt d'Enseignement et de Recherche (FER) au profit de l'Université de Dschang. Cette forêt qui avait été réservée depuis 1985 et qui n'a finalement vu son processus de classement aboutir qu'en 2016 est sujette à de

\section{MATERIEL ET METHODES}

Présentation de la zone d'étude : L'étude s'est réalisée de mars à juin 2017 dans la Forêt d'Enseignement et de Recherche (FER) de l'Université de Dschang. Ce massif forestier d'une superficie de 2400 ha est situé dans le département de Lom et Djerem, Région de l'Est- nombreuses pressions anthropiques dont l'exploitation du bois et l'agriculture. L'activité agricole occupait en 2016, 284 ha de la superficie de cette forêt soit $11,82 \%$ avec une nette dominance des SAFs cacaoyers occupant environ 76ha (Ndoh Nyemble, 2016). Pour éviter des conflits entre I'Université de Dschang et la population riveraine, il a été autorisé aux propriétaires des systèmes agroforestiers de la FER de continuer à entretenir leurs plantations existantes à condition de ne pas créer de nouvelles. Dans le cadre de l'aménagement de la FER, il est important de prendre en compte ces systèmes agroforestiers et de trouver des stratégies pour répondre à la fois au besoin de conservation des espèces et à l'amélioration des conditions de vie des ménages. D'où la nécessité de connaitre la composition et la structure actuelle, ainsi que les services écosystémiques rendus par ces SAFs. Cette étude a pour objectif d'évaluer la diversité ligneuse et les stocks de carbone des systèmes agroforestiers cacaoyers dans la FER de l'Université de Dschang à l'Est Cameroun.

Cameroun entre les latitudes $4^{\circ} 55^{\prime}-4^{\circ} 61^{\prime} \mathrm{N}$ et les longitudes $13^{\circ} 25^{\prime}-16^{\circ} 28^{\prime} E$ (figure 1). Elle a été classée en 2016, au profit de l'Université de Dschang pour des besoins de recherche.

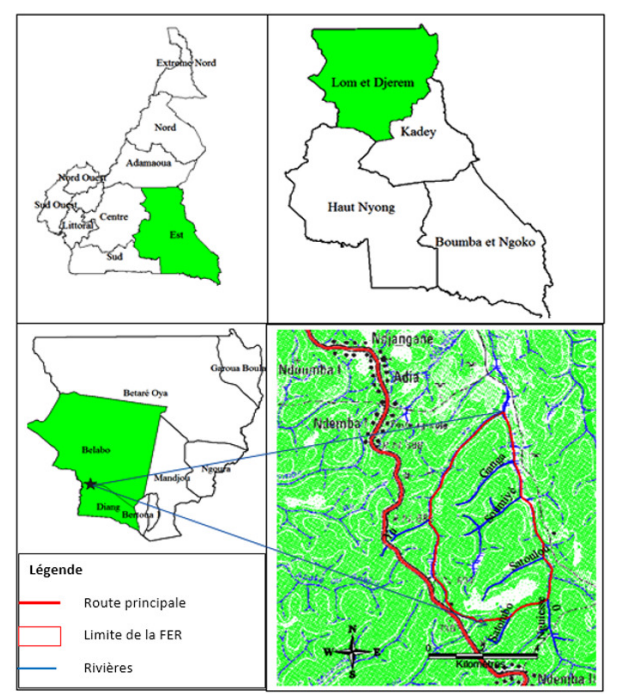

Figure 1 : Localisation de la région de L'Est et de la zone d'étude 
Le relief de la région est assez diversifié et très peu accidenté, l'altitude varie entre 500 et 800 mètres. Le climat est du type équatorial à 4 saisons. La température moyenne oscille entre 22 et $25^{\circ} \mathrm{C}$. La pluviométrie moyenne annuelle est de $1550 \mathrm{~mm}$. La FER est située dans la région floristique guinéo-congolaise, dans le domaine de la forêt dense humide semi-caducifoliée (Letouzey, 1985). La végétation est dominée par les familles de Sterculiaceae et Ulmaceae. Cette forêt renferme de nombreuses espèces commerciales: Terminalia superba, Triplochiton scleroxylon, Mansonia altissima, de nombreuses Meliaceae et des formations secondaires de Musanga et Albizia. Dans la partie Sud de la réserve s'étend une savane herbeuse à Imperata cylindrica parsemée d'arbustes du genre Annona. La partie ouest de la FER présente une zone dégradée occupée par des champs agricoles des populations des villages Ndemba 1 et Ndemba 2 qui y cultivent maïs, plantain, macabo, manioc, café et cacao. La FER est arrosé par plusieurs rivières permanentes. Les sols de la zone sont en majorité ferralitiques, argilo sablonneux ou argilo limoneux que l'on retrouve généralement dans les plaines avec par endroit une curasse latéritique. On note également la présence des sols hydromorphes rencontrés dans les bas-fonds, riches en matière organique.

Collecte des données : L'inventaire a été effectué dans tous les 21 Systèmes agroforestiers (SAFs) cacaoyers présents dans la FER. Dans chaque cacaoyère la mise en place des placettes de comptage a été faite en adaptant la méthode décrite par Harriah et al. (2011) avec l'installation des placettes principales de forme rectangulaire de $2400 \mathrm{~m}^{2}(40 \mathrm{~m} \times 60 \mathrm{~m})$ pour le comptage des ligneux ayant un diamètre à hauteur de poitrine supérieur ou égal à $30 \mathrm{~cm}$ et des placettes secondaires de $800 \mathrm{~m}^{2}(20 \mathrm{~m} \times 40 \mathrm{~m})$ pour les ligneux ayant un diamètre compris entre $5 \mathrm{~cm}$ et $30 \mathrm{~cm}$. Au total, 31 placettes principales ont été installées. L'inventaire a pris en compte la composition floristique (différentes espèces présentes dans les parcelles) et le diamètre à hauteur de poitrine. Les pieds de cacaoyers ont été également comptés et les diamètres mesurés. L'identification des espèces a été basée sur les noms vernaculaires en langue Pol. Les correspondances avec les noms scientifiques ont été établies à partir des manuels d'identification de Vivien et Faure (2012). Les âges des plantations, les usages et l'origine des arbres (conservés ou introduits) ont été renseignés par les propriétaires. Les plantations étaient âgées de 0 à 21 ans et ont été regroupées en quatre classes d'âge : $\leq 5$ ans, $5-10$ ans, $10-15$ ans et $>15$ ans. La superficie moyenne des plantations est de 3,5 ha. Le nombre de parcelles par classe d'âge variait entre 6 et 10 .

Analyse des données: La richesse spécifique, les indices de diversités de Shannon et de Simpson, l'équitabilité de piélou, les usages des arbres, les stocks de carbone ont été considérés comme les principaux paramètres de recherche.

La richesse spécifique correspond au nombre d'espèces que compte une communauté ou un peuplement (Ramade, 1994). Le nombre de famille et le nombre d'espèces présentes dans chaque parcelle a été déterminé ainsi que le nombre d'individus de chaque espèce.

Indice de diversité de Shannon-Weaver $\left(\mathrm{H}^{\prime}\right)$ permet de mesurer les possibilités d'interaction entre les espèces qui composent une communauté. Cet indice tient compte du nombre d'espèces présentes et de la répartition des individus au sein de ces espèces.

$\mathbf{H}^{\prime}=-\sum(\mathbf{n i} / \mathbf{N}) \log _{7}(\mathbf{n i} / \mathbf{N})(1)$

Où : ni est le nombre d'individus d'une espèce $\mathrm{i}$

; N le nombre total d'individus de toutes les espèces.

Indice d'équitabilité de Pielou (E) exprime la régularité, la répartition équitable des espèces au sein d'une communauté. Cet indice qui varie de 0 à 1 est maximal quand les espèces ont des abondances identiques dans le peuplement et il est minimal quand une seule espèce domine tout le peuplement.

\section{$\mathbf{E}=\mathbf{H}^{\prime} / \log _{2} \mathbf{S}(2)$}

$S$ est le nombre total d'espèces relevées et $H^{\prime}$ l'indice de Shannon.

L'indice de diversité de Simpson (D'): c'est la probabilité pour que deux individus tirés au hasard soient d'espèces différentes. II est représenté par la réciproque de l'indice de Simpson (D). Le maximum de diversité est représenté par la valeur 1 et le minimum de diversité par la valeur 0 (Danais, 1982).

$$
\mathbf{D}^{s}=\mathbf{1}-\mathbf{D}=\mathbf{1}-\mathbf{\Sigma}(\mathbf{n i} / \mathbf{N})^{2} \text { (3) }
$$

Où : ni est le nombre d'individus d'une espèce $\mathrm{i}$

; N le nombre total d'individus de toutes les espèces et $D$ I'Indice de Simpson.

Densité des espèces : La densité d'une espèce est le nombre d'individus de cette espèce à l'hectare. Elle est évaluée par la formule

$N=n / S(4)$

Avec : N la densité (en tiges/ha), 
$\mathrm{n}$ le nombre de tiges présentes sur la surface considérée et $S$ la surface considérée (ha).

\section{Estimation de la biomasse}

L'estimation de la biomasse aérienne des arbres associés et des cacaoyers s'est faite en utilisant l'équation allométrique de Chave et al. (2014).

$A G B=0,0673 \times\left(\rho D^{2} H\right)^{0,976}$

Où: AGB est la biomasse aérienne de l'arbre (en $\mathrm{kg}$ )

; $D$ est le diamètre de l'arbre $(\mathrm{en} \mathrm{cm})$

; $\mathrm{H}$ la hauteur totale de l'arbre (en $\mathrm{m}$ )

$; \rho$ la densité spécifique de l'arbre en $\left(\mathrm{g} \cdot \mathrm{cm}^{-3}\right)$.

Les densités spécifiques des espèces données par Zanne et al. (2009) et Vivien et Faure (2012) ont été utilisées.

L'estimation de la biomasse racinaire des ligneux sur pied s'est conformée aux lignes directives établies par GIEC (2006). Selon ces dernières, l'équivalence en biomasse racinaire des ligneux sur pied est trouvée en

\section{RESULTATS}

Richesse spécifique et diversité des SAFs cacaoyers : Dans l'ensemble, le cortège végétal des SAFs étudiés fait état de 71 espèces ligneuses multipliant la valeur de la biomasse aérienne (AGB) par un coefficient $R$ dont la valeur est estimée à 0,24.

$B G B=A G B \times R \quad(6)$

Où : $B G B$ est la biomasse souterraine

; $A G B$ la biomasse aérienne et $\mathrm{R}$ le ratio racine/tige.

Évaluation des stocks de carbone: Le stock de carbone a été obtenu en multipliant la somme des biomasses (aérienne et souterraine) par le ratio $\mathrm{CF}$ (carbone fraction) qui est de 0,47 (GIEC, 2006).

$C=(A G B+B G B) \times 0,47 \quad(7)$

Où : $C$ est le stock de carbone total, AGB la biomasse aérienne et $B G B$ la biomasse souterraine.

Analyses statistiques : Les données ont été analysées avec le logiciel Graphpad InStat, 2000. Les paramètres de richesse spécifique, les indices de diversités, et les stocks de carbone ont été soumis à une analyse de variance (ANOVA) et lorsque les différences étaient significatives entre les différentes classes d'âge, les moyennes étaient séparées par le test le test de StudentNewman-Keuls au seuil de $5 \%$.

appartenant à 32 familles. Le tableau 1 présente les caractéristiques des SAFs par classe d'âge.

Tableau 1 : Richesse spécifique et indices de diversité des différentes cacaoyères de la FER

\begin{tabular}{l|l|l|l|l|l|l}
\hline Classe d'Age & $\begin{array}{l}\text { Nombre de de } \\
\text { parcelles }\end{array}$ & $\begin{array}{l}\text { Nombre de } \\
\text { familles }\end{array}$ & $\begin{array}{l}\text { Nombre } \\
\text { d'espèces }\end{array}$ & Shannon & Piélou & Simpson \\
\hline$\leq 5$ ans & 11 & 30 & $46 \pm 20 \mathrm{a}$ & $3,35 \pm 0,84 \mathrm{a}$ & $0,73 \pm 0,06 \mathrm{~b}$ & $0,79 \pm 0,12 \mathrm{~b}$ \\
$5-10$ ans & 8 & 20 & $31 \pm 7 \mathrm{ab}$ & $3,30 \pm 0,49 \mathrm{a}$ & $0,91 \pm 0,03 \mathrm{a}$ & $0,89 \pm 0,02 \mathrm{a}$ \\
$10-15$ ans & 6 & 18 & $24 \pm 5 \mathrm{~b}$ & $3,19 \pm 0,36 \mathrm{a}$ & $0,71 \pm 0,04 \mathrm{~b}$ & $0,78 \pm 0,09 \mathrm{~b}$ \\
$<15$ ans & 6 & 18 & $30 \pm 5 \mathrm{ab}$ & $3,10 \pm 0,92 \mathrm{a}$ & $0,83 \pm 0,08 \mathrm{ab}$ & $0,83 \pm 0,09 \mathrm{ab}$ \\
FER & $\mathbf{3 1}$ & $\mathbf{3 2}$ & $\mathbf{7 2}$ & & & \\
\hline
\end{tabular}

Sur une même colonne, les valeurs suivies par une même lettre ne sont pas significativement au seuil de $5 \%$

Le tableau 1 montre que le nombre d'espèces varie de 24 à 46 espèces. La plus grande richesse spécifique a été observée dans les cacaoyères ayant un âge inférieur ou égal à 5 ans. La plus faible valeur de richesse spécifique a été observée dans les cacaoyères âgées entre 10 et 15 ans (24 espèces). Les indices de diversité de Shannon varient de 3,10 à 3,35 bits. II n'existe pas de différence significative entre les indices de diversité de Shannon pour les cacaoyères de classe d'âge différents. Cependant il existe une différence significative pour les indices de Simpson et d'équitabilité de Pielou. Les forts indices de diversité montrent que les individus sont bien répartis entre les espèces. La figure 2 présente le pourcentage des espèces par famille. 


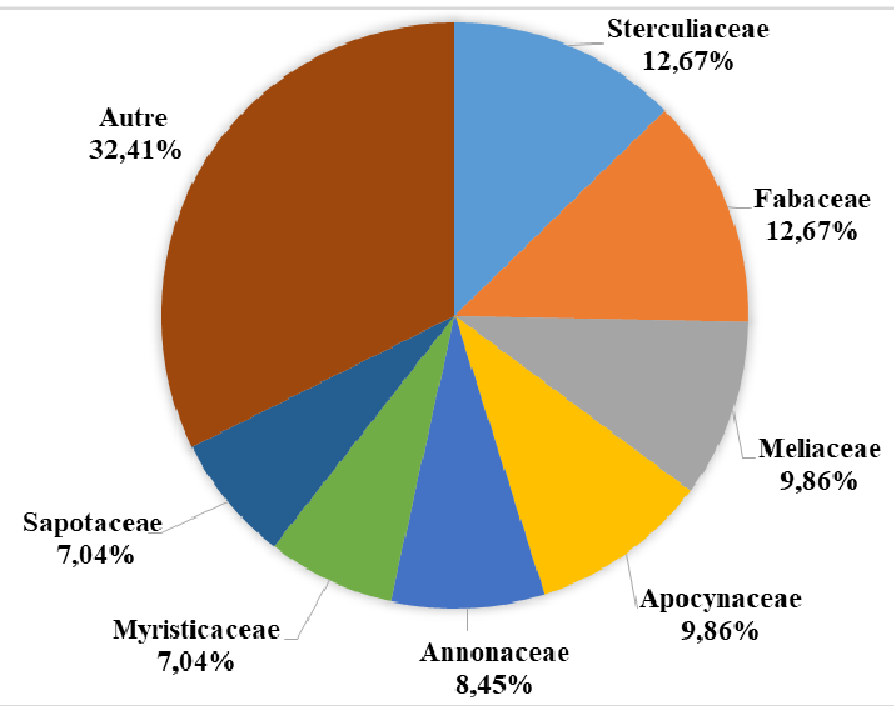

Figure 2 : Proportion des familles les plus représentées en nombre d'espèces

Sept familles sur les 32 représentent $67,6 \%$ des espèces totales recensées. Les familles les plus représentées sont les Sterculiaceae et les Fabaceae avec $12,67 \%$ des espèces chacune, suivies des Meliaceae et
Apocynaceae, avec 9,86\% chacune. Le terme « autres » représente les 26 autres familles qui ont chacune moins de $5 \%$ des espèces recensées. L'abondance en termes du nombre d'individus est présentée par la figure 3 .

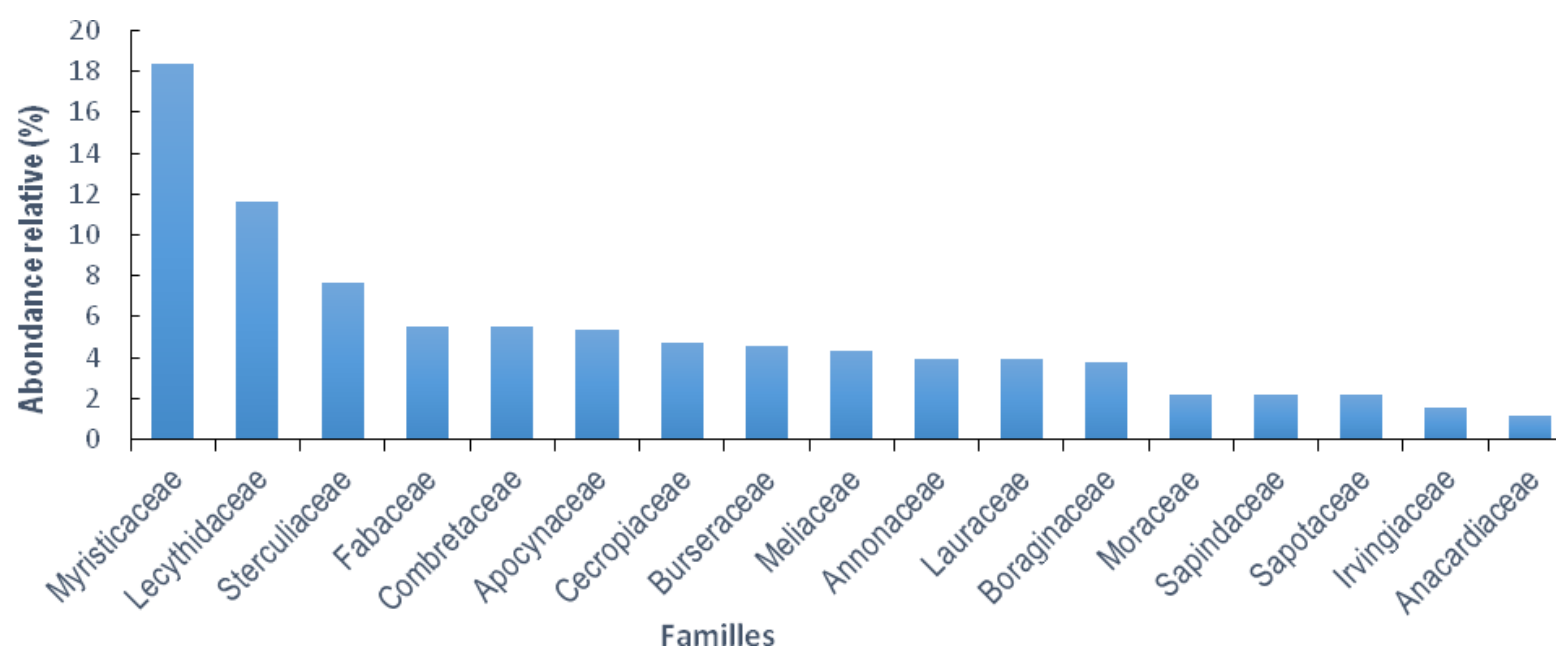

Figure 3 : Abondance relative des familles ayant plus de $1 \%$ des individus

La famille des Myristicaceae est la famille la plus représentée en nombre d'individus (18,34\%). Elle est suivie des Lecythidaceae $(11,63 \%)$ et Sterculiaceae $(7,69 \%)$. Douze familles ont une abondance relative inférieure à $1 \%$. L'espèce la plus abondante dans les cacaoyers de la FER est Pycnanthus angolensis avec
$16,56 \%$ des individus (figure 4), suivie de Petersianthus macrocarpus (12\%), Musanga cecropioides (5,33\%) et Terminalia superba (4,90\%), Persea americana et Cordia platythyrsa $(3,75 \%)$. Cinquante-sept espèces ont chacune moins de $2 \%$ d'abondance relative. 


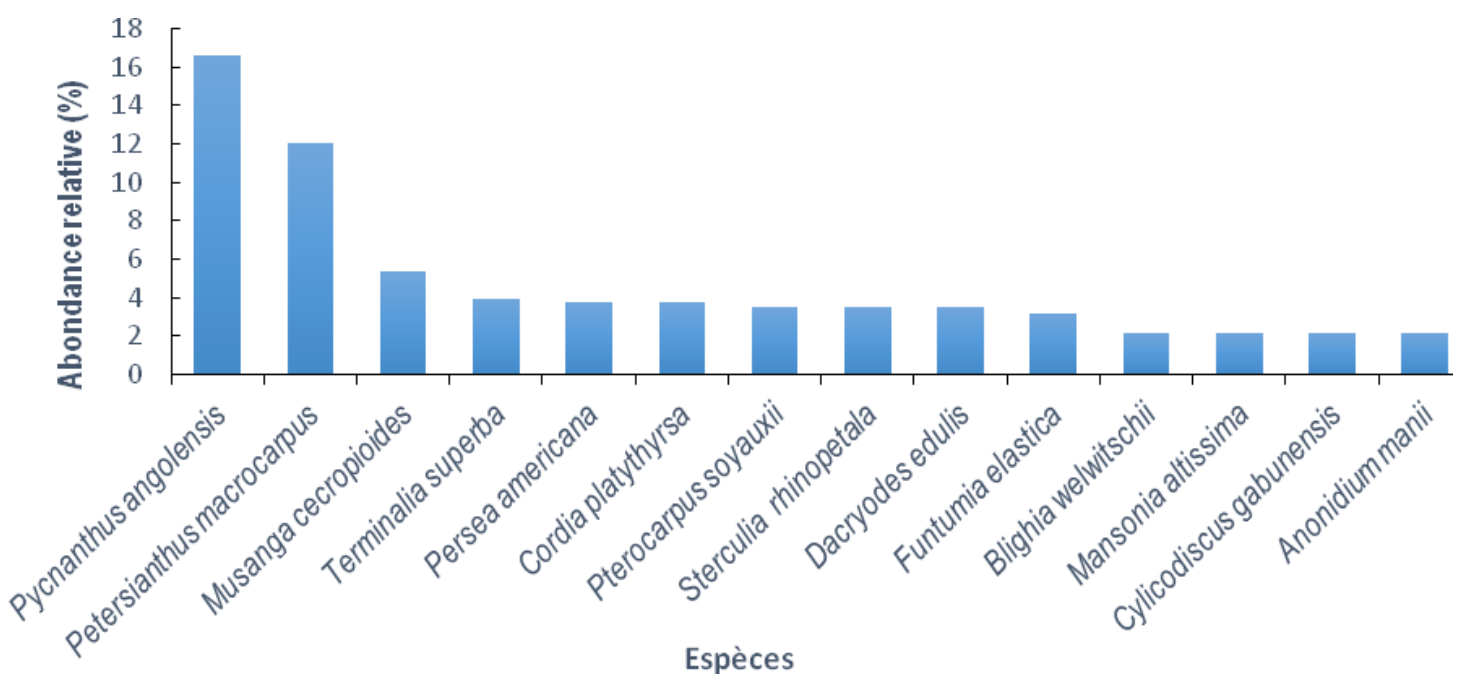

Figure 4 : Espèces associées aux cacaoyers ayant plus de $2 \%$ d'abondance relative

Densité des arbres associés aux cacaoyers: La proportion des arbres introduits est de $9,34 \%$ du total des arbres inventoriés tandis que les conservés lors de la mise en culture de la cacaoyère représentent $90,65 \%$. Les espèces les plus introduites sont par ordre d'importance Persea americana (Lauraceae), Dacryodes edulis (Burseraceae), Mangifera indica (Annonaceae),
Baillonella toxisperma (Sapotaceae) et Irvingia gabonensis (Irvingiaceae). Les espèces les plus conservées sont Pycnanthus angolensis (Myristicaceae), Petersianthus macrocarpus (Lecythidiaceae) et Terminalia superba (Combretaceae). Le tableau 2 présente les densités de ces arbres associés.

Tableau 2 : Densité des arbres associés dans les cacaoyères de la FER

\begin{tabular}{l|l|l}
\hline \multirow{2}{*}{ Classe d'âge des cacaoyers } & \multicolumn{2}{|c}{ Arbres associés } \\
\cline { 2 - 3 } & Arbres introduits & Arbres conservés \\
\hline$\leq 5$ ans & $24,30 \pm 22$ a & $147,57 \pm 53 a$ \\
\hline $5-10$ ans & $24,43 \pm 20 a$ & $145,52 \pm 10 a$ \\
\hline $10-15$ ans & $22,65 \pm 19 a$ & $131,94 \pm 18 a$ \\
\hline$>15$ ans & $23,27 \pm 7 a$ & $142,21 \pm 18 a$ \\
\hline
\end{tabular}

Sur une même colonne, les valeurs suivies par une même lettre ne sont pas significativement différentes au seuil de $5 \%$

Pour toutes les classes d'âges des cacaoyères, le nombre d'arbres associés conservés est supérieur au nombre d'arbres introduits. II existe une différence significative entre le nombre d'arbres associés introduits et le nombre d'arbres conservés dans les différentes cacaoyères. II existe une différence hautement significative entre le nombre d'arbres associés introduits et le nombre d'arbres conservés dans les différentes cacaoyères $(F=15,83$ et $P=0,016)$. Cependant aucune différence significative de densité des arbres associés aux cacaoyers n'est mise en évidence entre les différentes classes d'âge.

Usages des arbres associés aux cacaoyers: La conservation ou l'introduction dans les SAFs d'un grand nombre d'espèces forestières et fruitières répond en grande partie aux différents besoins des ménages. Les enquêtes ont permis d'identifier cinq usages pour les arbres associés aux cacaoyers en dehors de l'ombrage. Parmi ces espèces, $25,35 \%$ ont un seul usage (figure 5) ; $38,03 \%$ des espèces ont deux usages et $9,86 \%$ en ont trois. $26,76 \%$ des espèces n'avaient aucun usage à l'exemple de Musanga cecropioides et Picralima nitida. A l'exception de Musanga cecropioides, les cinq espèces les plus abondantes (Pycnanthus angolensis, Petersianthus macrocarpus, Persea americana et Terminalia superba) ont au moins deux usages chacun, ce qui confirme le caractère multi-usage de ces espèces. 


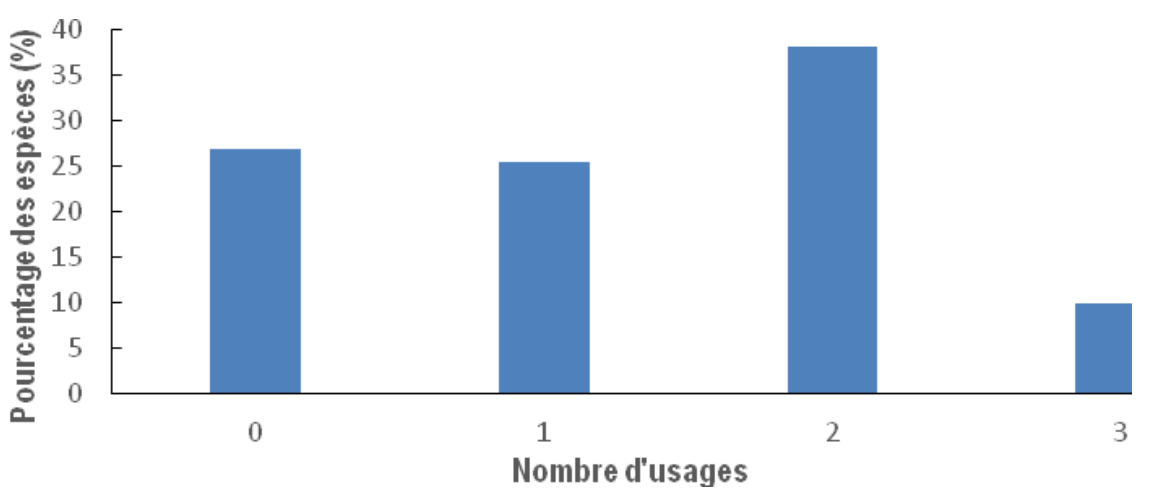

Figure 5 : Répartition des espèces associées aux cacaoyers en fonction du nombre d'usages

En ce qui concerne les catégories d'usages (figure 6), $47,8 \%$ des arbres fournissent du bois, $32 \%$ offrent des produits médicinaux, $18,30 \%$ des produits alimentaires,
$9,85 \%$ améliorent ou maintiennent la fertilité du sol et $2,80 \%$ ont un rôle culturel.

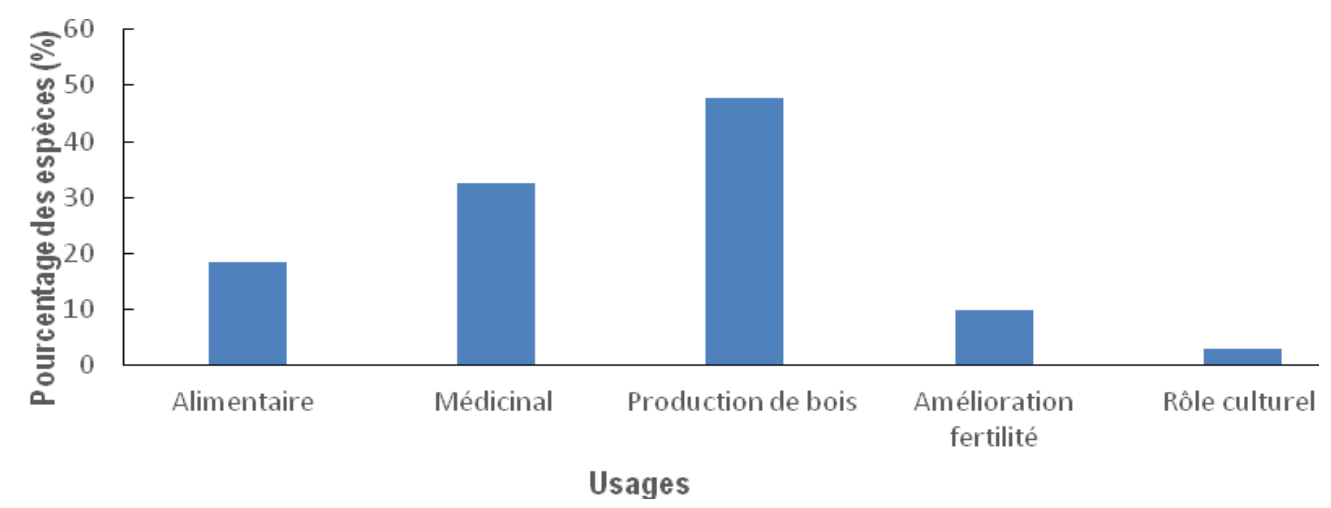

Figure 6 : Usages des espèces associées aux cacaoyers

Les arbres tiennent une place importante dans la stratégie de sécurisation alimentaire, notamment par l'utilisation des graines (Irvingia gabonensis, Ricinodendron heudelotii, Baillonnella toxisperma), des feuilles (Ceiba pentandra) et des fruits (Dacryodes edulis, Persea americana, Mangifera indica) dans l'alimentation des populations de la zone. Les produits alimentaires sont en majorité destinés à la consommation des ménages et seul l'excédent est vendu. Les populations font recours aux arbres dans la pharmacopée traditionnelle en raison de la très faible couverture sanitaire dans la zone. Les organes utilisés sont les feuilles, l'écorce et les racines. L'amélioration de la fertilité des sols constitue un apport des arbres dans les champs et cela guide les paysans dans le choix des espèces à gérer. Les paysans reconnaissent que certaines espèces ligneuses à l'exemple de Pycnanthus angolensis et Ceiba pentandra améliorent la fertilité des sols. La production du bois est un des rôles importants des ligneux qui motive les paysans à entretenir et protéger les arbres dans leurs SAFs. Certaines de ces essences de bois d'œuvre (Entandrophragma cylindricum, Milicia excelsa, Petersianthus macrocarpus, Triplochiton scleroxylon) sont également des hôtes de chenilles comestibles.

Contraintes à l'introduction des arbres dans les SAFs cacaoyers : Les enquêtes ont révélé qu'il existe plusieurs freins à l'introduction des arbres dans les SAFs cacaoyers (figure 7). Les principales contraintes sont l'excès d'ombrage cités par $76,2 \%$ des agriculteurs, le manque de semences et de plants $(71,42 \%)$, la non maitrise des techniques sylvicoles $(66,67 \%)$, la lente 
croissance des arbres (57\%) et l'éloignement des villages de la FER $(52,28 \%)$.

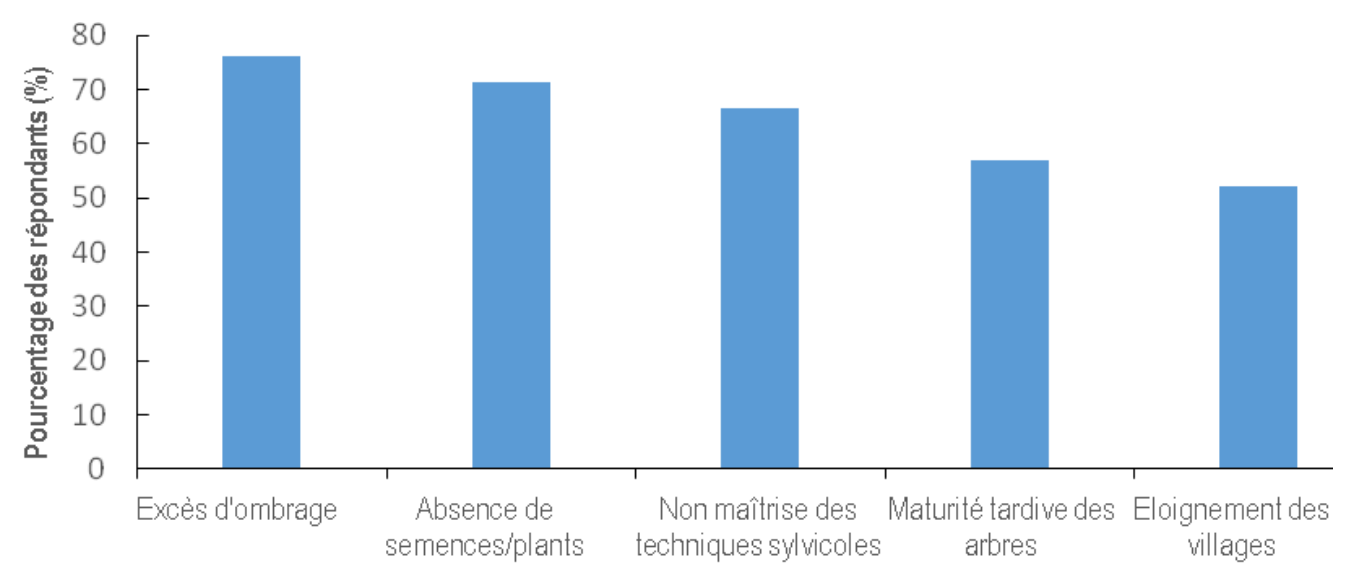

Contraintes

Figure 7: Principales contraintes à la plantation des arbres dans les SAF Cacaoyers de la FER

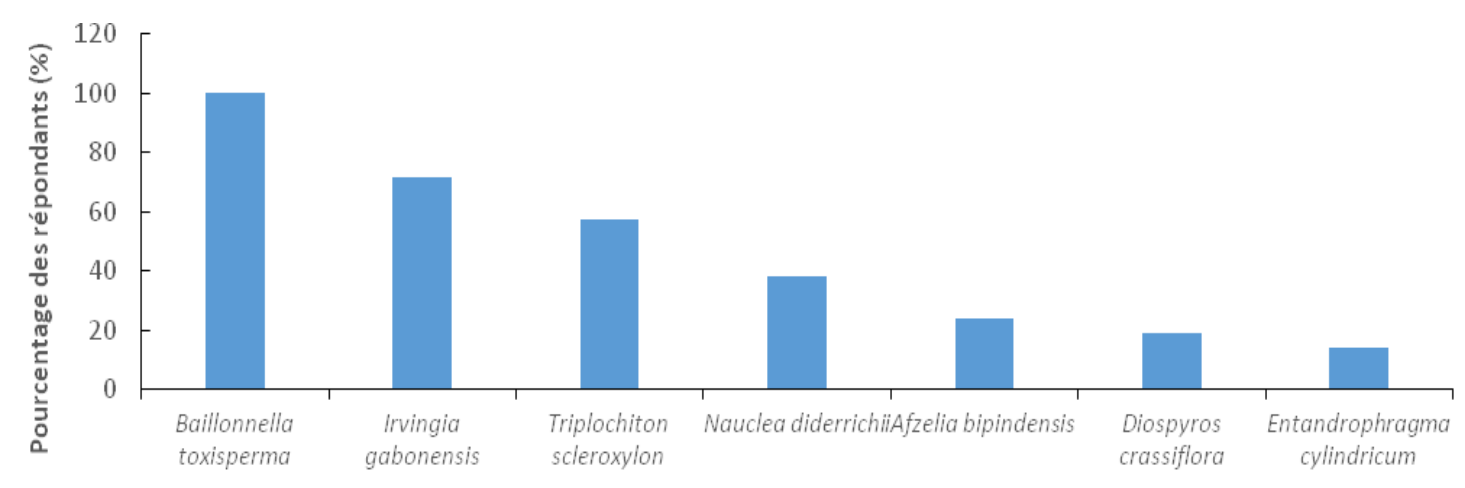

Espèces sollicitées

Figure 8 : Espèces d'arbres que les agriculteurs souhaiteraient introduire dans leur SAFs

Malgré ces contraintes, les agriculteurs ont exprimé leur volonté d'introduire des espèces spécifiques dans leurs parcelles. Ces espèces sont présentées à la figure 8 . II ressort de la figure 8 que le Moabi (Baillonella toxisperma) est l'espèce la plus sollicitée. Ceci se justifie surtout par le fait qu'il s'agit d'une essence très précieuse, utile aussi bien pour son bois que pour la pharmacopée et l'alimentation humaine. L'Andok (Irvingia gabonensis) est également très sollicité en raison de ses graines utilisées dans l'alimentation et dont l'importance socio-économique est avérée pour les populations. Les autres espèces sollicitées Triplochiton scleroxylon, Nauclea diderrichii, Afzelia bipindensis, Diospyros crassiflora, Entandrophragma cylindricum sont des espèces de bois d'œuvre.

Stocks de carbone des cacaoyers et des arbres associés: La quantité de carbone stocké par les cacaoyers et les arbres associés a été évaluée et les résultats sont consignés dans le tableau 3. 
Tableau 3: Stocks de carbone des cacaoyers et des arbres associés dans les SAFs de la FER

\begin{tabular}{lllll}
\hline \multirow{2}{*}{ Classe d'âge } & \multicolumn{3}{l}{ Stocks de carbone $(\mathrm{tC} / \mathrm{ha})$} & \\
\cline { 2 - 5 } & Cacaoyers & $\begin{array}{l}\text { Arbres associés } \\
\text { Arbres Dhp }<\mathbf{3 0} \mathbf{c m}\end{array}$ & Arbres Dhp $\geq \mathbf{3 0}$ & Total \\
\hline$\leq 5$ ans & $0,87 \pm 0,10 \mathrm{c}$ & $16,15 \pm 8,19 \mathrm{a}$ & $110,3 \pm 35,41 \mathrm{a}$ & $127,32 \pm 43,70 \mathrm{a}$ \\
$5-10$ ans & $1,32 \pm 0,19 \mathrm{~b}$ & $11,627 \pm 3,54 \mathrm{a}$ & $76,202 \pm 32,17 \mathrm{a}$ & $89,15 \pm 35,90 \mathrm{a}$ \\
$10-15$ ans & $2,33 \pm 0,48 \mathrm{ab}$ & $14,38 \pm 40 \mathrm{a}$ & $82,11 \pm 26,3 \mathrm{a}$ & $98,82 \pm 30,82 \mathrm{a}$ \\
$>15$ ans & $3,89 \pm 1,98 \mathrm{a}$ & $10,02 \pm 8,64 \mathrm{a}$ & $99,73 \pm 35,64 \mathrm{a}$ & $113,64 \pm 38,20 \mathrm{a}$ \\
\hline
\end{tabular}

Sur une même colonne, les valeurs suivies par une même lettre ne sont pas significativement différentes au seuil de $5 \%$

Les stocks de carbone des cacaoyers est significativement différents de celui des arbres associés ( $F=14,39$ et $P=0,0002)$. Les cacaoyers stockent moins de carbone que les arbres associés avec une moyenne de $2,25 \pm 0,66 \mathrm{tC} / \mathrm{ha}$. La quantité de carbone séquestrée par les cacaoyers augmente avec l'âge de la cacaoyère. On note une différence significative entre les différentes classes d'âge. Cette différence est liée au diamètre et à la surface terrière des cacaoyers qui augmentent avec l'âge. La quantité de carbone stocké par les arbres associés varie de $89 \mathrm{tC} /$ ha pour les SAFs âgés de 5 à 10 ans à $127 \mathrm{tC} /$ ha pour ceux ayant un âge inférieur ou égal à 5 ans. Le stock de carbone des arbres associés est différemment reparti entre les arbres de diamètre supérieur à $30 \mathrm{~cm}$ et ceux dont le diamètre est inférieur à
$30 \mathrm{~cm}$. En effet, les arbres de plus de $30 \mathrm{~cm}$ de diamètre sont ceux qui contribuent le plus au carbone total séquestré. La figure 9 présente les familles taxonomiques ayant les plus fortes proportions de carbone stocké. La famille des Myristicaceae est celle qui stocke le plus de carbone (15\% du carbone des arbres associés). Elle est suivie des Lecythidaceae (14\%) et des Fabaceae (12\%). Des espèces conservées, Petersianthus macrocarpus est celle qui stocke le plus de carbone avec une moyenne de 9,44 tC/ha suivie de Terminalia superba (8,54 tC/ha) et Pycnanthus angolensis (6,35 tC/ha). Dans la catégorie des arbres introduits, Persea americana est l'espèce qui a la plus grande quantité de carbone stocké $(1,82 \mathrm{tC} / \mathrm{ha})$, suivie de Mangifera indica (1,3 tC/ha) et Dacryodes edulis $(1,13 \mathrm{tC} / \mathrm{ha})$.

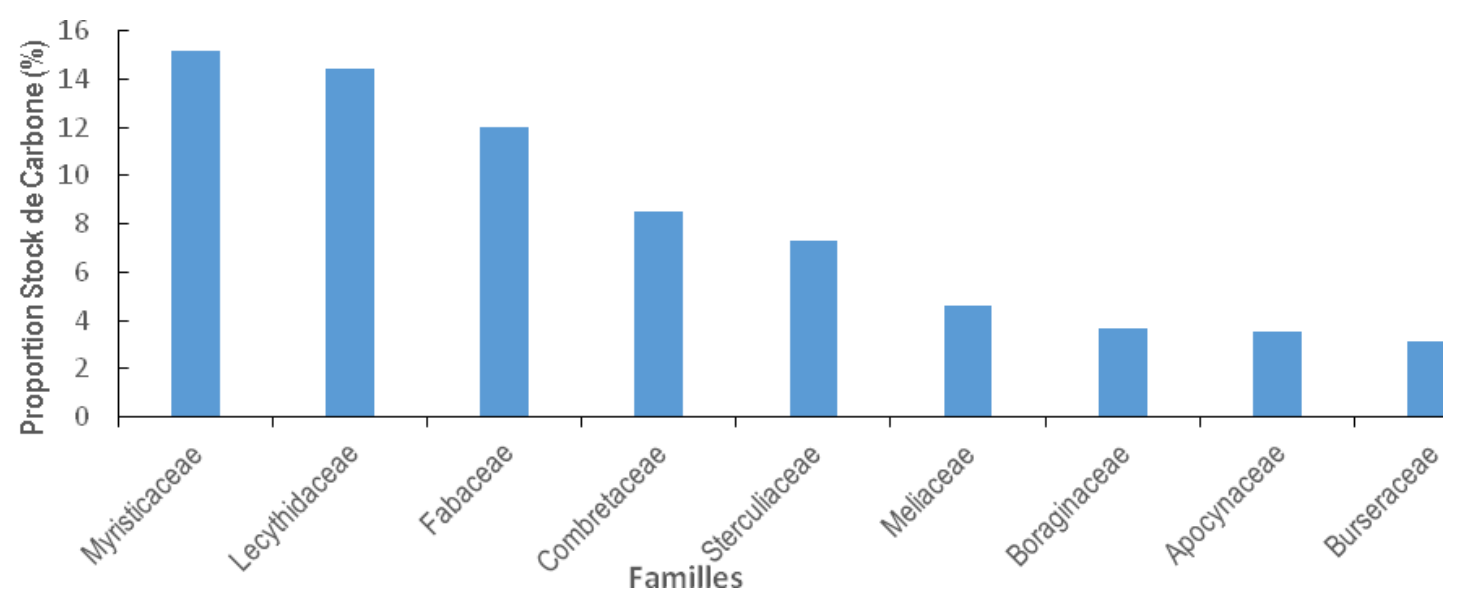

Figure 9 : Groupes taxonomiques ayant les plus grandes proportions de stock de carbone

\section{DISCUSSION}

Les systèmes agroforestiers cacaoyers présents dans la forêt d'enseignement et de recherche de l'Université de Dschang ont un âge compris entre 0 et 21 ans avec une dominance de jeunes cacaoyères d'âge inférieur à 10 ans. Ces SAFs sont relativement jeunes comparés aux autres cacaoyères où des études similaires ont été menées au Cameroun et dont l'âge variait de 0 à plus de 40 ans (Jagoret, 2011 ; Jagoret et al., 2011 ; Saj et al., 2013). Soixante-douze espèces ont été inventoriées dans ces SAFs. Cette richesse spécifique est semblable 
à celle trouvée par Jagoret et al. (2014a) dans les sites de Bokito (70 espèces), Zima (64 espèces) ; Ngomedzap (84 espèces) au Centre Cameroun. Les indices de diversité de Shannon sont supérieurs à 3 pour toutes les classes d'âge indiquant une grande diversité et une bonne répartition en espèce des cacaoyères de la FER. Ces indices sont similaires à ceux trouvés par Sonwa et al. (2007) dans les cacaoyères de la zone de forêt dense humide du Sud Cameroun et supérieurs à ceux trouvés par Jagoret et al. (2011) dans la zone de transition forêtsavane du centre Cameroun. La densité des arbres associés aux cacaoyers (166 arbres/ha) est supérieure à celle trouvée par Norgrove et Hauser (2013) dans les cacaoyères de Zoatoupsie au Sud Cameroun (115 arbres/ha). Elle est cependant inférieure à celle de 233 arbres/ha obtenue par Saj et al. (2013) à Ngomedzap au centre Cameroun. Cette différence est principalement due au nombre d'arbres introduits qui est inférieur dans notre site d'étude (23 arbres/ha) contre 83 arbres/ha à Ngomedzap. Le nombre d'arbres conservés est sensiblement le même dans les deux zones (143 et 150 tiges/ha). En effet, dans notre site d'étude, en raison de la faible densité de population et l'éloignement des grands marchés, le besoin local en espèces fruitières comestibles semble moins élevé que dans la région du Centre. Les paysans accordent une valeur particulière à certaines espèces qu'ils conservent volontairement $(91 \%)$ et introduisent $(9 \%)$ dans leurs SAFs Cacaoyères. Les espèces fruitières Persea americana, Dacryodes edulis et Mangifera indica sont les espèces les plus introduites en raison de leur valeur alimentaire. Les espèces forestières couvrent les autres usages: bois de chauffe et bois d'œuvre, produits médicinaux et maintien de la fertilité du sol. Certaines de ces espèces comme Entandrophragma cylindricum Irvingia gabonensis, Triplochiton scleroxylon, ... sont également connues pour être des hôtes d'espèces de chenilles comestibles (Lisongo et al., 2010 ; Okangola, 2016). Le palmier à huile (Elaeis guineensis) est très faiblement introduit dans notre zone d'étude contrairement à ce qui a été observé dans les régions du Sud et du Centre où il fait partie des espèces les plus introduites (Sonwa et al., 2007 ; Jagoret, 2014a). La majorité des espèces conservées sont des espèces de bois d'œuvre. Le bois des SAFs est principalement vendu

\section{CONCLUSION}

Les systèmes agroforestiers cacaoyers de la FER ont une grande richesse spécifique, avec une importante proportion d'arbres conservés. Les agriculteurs préservent des espèces forestières mais introduisent localement. Parmi les cinq espèces les plus exploitées au Cameroun (Eba'a Atyi et al., 2013), quatre ont été trouvées dans les SAFs suggérant que le bois provenant de ces derniers pourrait avoir un marché intéressant. Toutefois, avec le classement du massif forestier en Forêt d'Enseignement et de Recherche, les agriculteurs ne pourront plus disposer librement de ces arbres comme par le passé. L'âge des cacaoyères n'a pas eu une incidence significative sur la quantité totale de carbone stocké. La plus grande partie du carbone est stocké par les arbres associés de diamètre supérieur à $30 \mathrm{~cm}$ et seulement une très faible proportion de carbone est stockée par les cacaoyers $(2,10 \%)$. La moyenne de stock de carbone de $107 \mathrm{tC} / \mathrm{ha}$ trouvée dans cette étude est similaire à celle trouvée par Gockowski et Sonwa (2010). Elle est cependant supérieure à celle trouvée par Saj et al. (2013) au centre Cameroun et inférieure aux résultats de Njomnang et al. (2011) et Norgrove et Hauser (2013) au Sud Cameroun. Ces différences sont probablement dues à la diversité des milieux et des densités des arbres mais également aux différentes équations allométriques utilisées. Les agriculteurs souhaiteraient planter certaines espèces qui ont une forte valeur alimentaire et de production du bois mais qui actuellement ont une faible abondance dans leurs SAFs. Cependant plusieurs contraintes telles que l'absence de plants et la non maîtrise des techniques sylvicoles, la lente croissance des arbres et l'excès d'ombrage, sont les freins à cette introduction. La rentabilité économique directement liée à l'action d'introduire des arbres dans les SAFs n'intervient que plusieurs années plus tard en raison de la lente croissance de ces derniers et les agriculteurs n'ont pas toujours cette patience. Bien que les cacaoyers nécessitent de l'ombrage, des études ont montré qu'un excès ombrage entraine une baisse des rendements (Gala et al., 2017) notamment parce que les plants de cacaoyers entrent en compétition avec les arbres pour la lumière et les éléments minéraux du sol. Plusieurs de ces contraintes à l'introduction des arbres citées par les agriculteurs peuvent être levées par une assistance à la production et à la distribution du matériel végétal de qualité et un renforcement des leurs capacités pour la plantation et la gestion des arbres.

aussi des arbres fruitiers à usage alimentaire, médicinal, de production de bois et d'amélioration de la fertilité du sol. Les SAFs de la FER contribuent significativement à la conservation des espèces ligneuses et au stockage de 
carbone. La combinaison du rôle écologique et économique de ces SAFs permettrait non seulement de protéger l'environnement mais aussi d'améliorer les conditions de vies des populations. Ainsi, les stratégies

\section{REFERENCES}

Atangana A, Khasa D, Chang S, Degrande A, 2014. Agroforesterie tropicale. Département de Sciences du bois et de la forêt, Université Laval. 412pp.

CARPE, 2005. The forest of the Congo Basin: A preliminary assessment. Central African Regional Program for the Environment (CARPE), 37pp.

Chave J, Réjou-Méchain M, Búrquez A, Chidumayo E., Colgan MS, Delitti WBC., Duque A, Eid T, Fearnside PM, Goodman RC, Henry M, Martínez-Yrízar A., Mugasha W, 2014. Improved allometric models to estimate the aboveground biomass of tropical trees. Global Change Biology 20: 3177-3190.

Danais M, 1982. La diversité en écologie: Analyse bibliographique. Botanica Rhedonica 17: 77-104.

Eba'a Atyi R, Lescuyer G, Ngouhouo JP, Fouda T, 2013. (Eds.), Étude de l'importance économique et social du secteur forestier et faunique au Cameroun. CIFOR, Bogor Indonésie. 315pp.

Ernst C, Mayaux $P$, Verhegghen A, Bodart C, Musampa C, Defourny $P$, 2013. National forest cover change in Congo Basin: deforestation, reforestation, degradation and regeneration for the years 1990, 2000 and 2005. Global Change Biology 19 (4): 1173-1187.

Gala J, Bohoussou Y, N'Dri, F, Akotto Odi, Ýao-Kouamé A, 2017. Impact des arbres associés sur l'exploitation cacaoyère dans les zones de transition forêt-savane : cas de M'Brimbo (Centre-Sud de la Côte d'Ivoire). European Scientific Journal 13 (1) : 164-181.

GIEC, 2006. Guide pour l'inventaire national des gaz à effet de serre ; agriculture, foresterie et autre usage des terres. Institute for Global Environnemental Strategies, Japon 4: 46-52.

Gockowski J. and Sonwa D, 2010. Cocoa intensification scenarios and their predicted impact on $\mathrm{CO}_{2}$ emissions, biodiversity conservation and rural livelihoods in the Guinea Rain Forest of West Africa. Environmental Management 48: 307-32, 2010.

Gockowski J, Tchatat M, Dondjang JP, Hietet G, Fouda $T, 2010$. An empirical analysis of the biodiversity d'amélioration doivent tenir compte des espèces sollicitées par les agriculteurs dans la recherche des meilleurs compromis entre la production cacaoyère, et les autres services rendus par les arbres.

and economic returns to cocoa agroforests in southern Cameroon. Journal of Sustainable Forestry $29: 638-670$.

Hairiah K, Dewi S, Agus F, Velarde S, Ekadinata A, Rahayu S and van Noordwijk M, 2010. Measuring carbon stocks across land use systems: A Manual. Bogor, Indonesia. World Agroforestry Centre (ICRAF), SEA Regional Office.

ICCO, 2017. Annual report 2014/2015. International Cocoa Organization, 76pp.

Jagoret $P, 2011$. Analyse et évaluation de systèmes agroforestiers complexes sur le long terme : Application aux systèmes de culture à base de cacaoyer au Centre Cameroun. Thèse de doctorat. Montpellier SupAgro, Montpellier. 288pp.

Jagoret $P$, Michel-Dounias, I, Malézieux, E, 2011. Longterm dynamics of cocoa agroforests: a case study in central Cameroon. Agroforestry Systems 81: 267-278.

Jagoret P, Kwesseu J, Messie C, Michel-Dounias I, Malézieux E, 2014a. Farmers' assessment of the use value of agrobiodiversity in complex cocoa agroforestry systems in central Cameroon, Agroforestry Systems 88: 983-1000.

Jagoret P, Kwesseu J, Messie C, Michel-Dounias I, Malézieux E, 2014b. Valeurs d'usage des ligneux utilisés en agroforesterie: les cacaoyères du Centre-Cameroun. Bois et Forêts des Tropiques $321:$ 45-54.

Letouzey R, 1985. Notice explicative de la carte phytogéographique du Cameroun au 1/500.000. Encyclopédie biologique 69, Edition Paul Le Chevalier, Paris. 240 pp.

Lisongo J, Wetsi JL, Ntahobavuka H, 2010. Enquête sur les chenilles comestibles et les divers usages de leurs plantes hôtes dans les districts de Kisangani et de la Tshopo (R.D.Congo). GeoEco-Trop 34 : 139-146.

Ndoh-Nyemble C, 2016. Caractérisation de la végétation de la forêt d'enseignement et de recherche du "bloc FASA Ndemba"de Belabo-Diang (EstCameroun) : contraintes et opportunités pour 
son classement. Mémoire de fin d'études. Université de Dschang 77pp.

Njomgang R, Yemefack M, Nounamo L, Moukam A, KottoSame J, 2011. Dynamics of shifting agricultural systems and organic carbon sequestration in Southern Cameroon. Tropicultura 29: 176-182.

Norgrove L. and Hauser S, 2013. Carbon stocks in shaded Theobroma cacao farms and adjacent secondary forests of similar age in Cameroon. Tropical Ecology 54 (1): 15-22.

Okangola E, Solomo E, Lituka Y, Tchatchambe WB, Mate M, Upoki A, Dudu A, Asimonyio J, Mpiana T, Koto-te-Nyiwa Ngbolua, 2016. Étude ethnobotanique et floristique de quelques plantes hôtes des chenilles comestibles à usage médicinal dans Province de la Tshopo, RD Congo. International Journal of Innovation and Scientific Research 26 (1): 146-160.

Ramade F, 1994. Éléments d'écologie. Écologie fondamentale 2. Ediscience international, 594pp.

Saj S, Jagoret P, Todem Ngogue H, 2013. Carbon storage and density dynamics of associated trees in three contrasting Theobroma cacao agroforests of Central Cameroon. Agroforestry Systems 87: 1309-1320.

Saj S, Durot C, Mvondo-Sakouma K, Tayo Gamo K, Avana-Tientcheu, M-L, 2017. Contribution of companion trees to long-term tree conservation, carbon storage and agroforest sustainability: a functional analysis of the diversity in cacao plantations of Central Cameroon. International Journal of Agricultural Sustainability 15: 282302.

Sonwa DJ, Nkongmeneck BA, Weise SF, Tchatat M, Adesina AA, Jansens MJJ, 2007. Diversity of plants in cocoa agroforests in the humid forest zone of Southern Cameroon. Biodiversity and Conservation 16: 2385-2400.

Sunderlin WD, Ndoye O, Bikié H, 2000. Economic crisis, farming systems and forest cover change in the humid forest zone of Cameroon. International Forestry Review 2: 173-181.

Vaast P. and Somarriba E, 2014. Trade-offs between crop intensification and ecosystem services: the role of agroforestry in cocoa cultivation. Agroforestry Systems 88 :947-956, 2014.

Vivien J. and Faure JJ, 2012. Arbres des forêts denses d'Afrique centrale. Espèces du Cameroun. Paris, France, Agence de coopération culturelle et technique. 954pp.

Zanne AE, Lopez-Gonzalez G, Coomes DA, llic J, Jansen S, Lewis SL, Miller RB, Swenson NG, Wiemann MC, Chave J, 2009. Data from: Towards a worldwide wood economics spectrum. Dryad Data Rep. 